\title{
MS020.P05
}

\section{Can substitution effect control the metal-ligand polymerization?!}

Ali Samie $^{1}{ }^{,}$Alireza Salimi $^{1}$, Jered C. Garrison ${ }^{2}$

${ }^{1}$ Department Of Chemistry, Ferdowsi University Of Mashhad, Mashhad 917751436, I.R., Mashhad, Iran, Islamic Rep., ${ }^{2}$ Department of Pharmaceutical Sciences, University of Nebraska Medical Center, 985830, USA, Nebraska, United States E-mail: alisamie.chemistry@gmail.com

On the base of our previous studies due to the presence of halides in our complexes there is strong probability for obtaining coordination polymers. Crystal engineering well-defined as the understanding of intermolecular interactions in the context of crystal packing and the utilization of such understanding in the design of new solids with desired physical and chemical properties. Owing to the fact that the small changes in the ligands may play a significant role in the complexes, the organic ligand (1-naphtyl pyridine-2-carboxylate) (L), was synthesized. In order make more steric repulsion around of the complexes resulted in the substitution effect around of the naphtyl moiety was hired in the design. Relocation in the position of functional group was fulfilled our expectations. Three new mercury (II) halide complexes, $[\mathrm{Hg}(\mathrm{L}) 2 \mathrm{Cl} 2](1),[\mathrm{Hg}(\mathrm{L}) 2 \mathrm{Br} 2](2)$ and $[\mathrm{Hg}(\mathrm{L}) 2 \mathrm{I} 2]$ (3) were synthesized. All of the compounds were fully characterized using FT-IR, TGA, DSC, mass spectrometry, CHNOS elemental analyses, PXRD, NMR and SCXRD. The results indicate that metal-ligand polymerization controlled by substitution effect. The coordination geometry around the $\mathrm{Hg}$ (II) ion is seesaw shape in distorted tetrahedral geometry for compounds (1), (2) and (3) with T4 of $0.74,0.76$ and 0.78 , respectively. Due to the replacement of the functional group (mentioned substitution effect), flexibility of coodinated ligand was decreased. In the previously reported structures, the angle between two planes of aromatic rings in the analogous ligand was 78.370 which changed to the $59.470,50.750$ and 64.90 o for mercury halide series complexes. In present study these angles are $89.09,89.73$ and 88.90 for titled complexes based on new designed ligand. Consequently, this study emphasize that the substitution effect can play the significant role through the flexibility of designed ligand to control the metal-ligand polymerization.

[1] Desiraju. G. R. et al. (2011) Crystal Engineering: A Textbook, World Scientific, Singapore.

[2] Merz, K. (2006) Cryst. Growth Des. 6, 1615-1619.

[3] Li, Y-X, et al. (2016) CrystEngComm.18, 2098-2104.
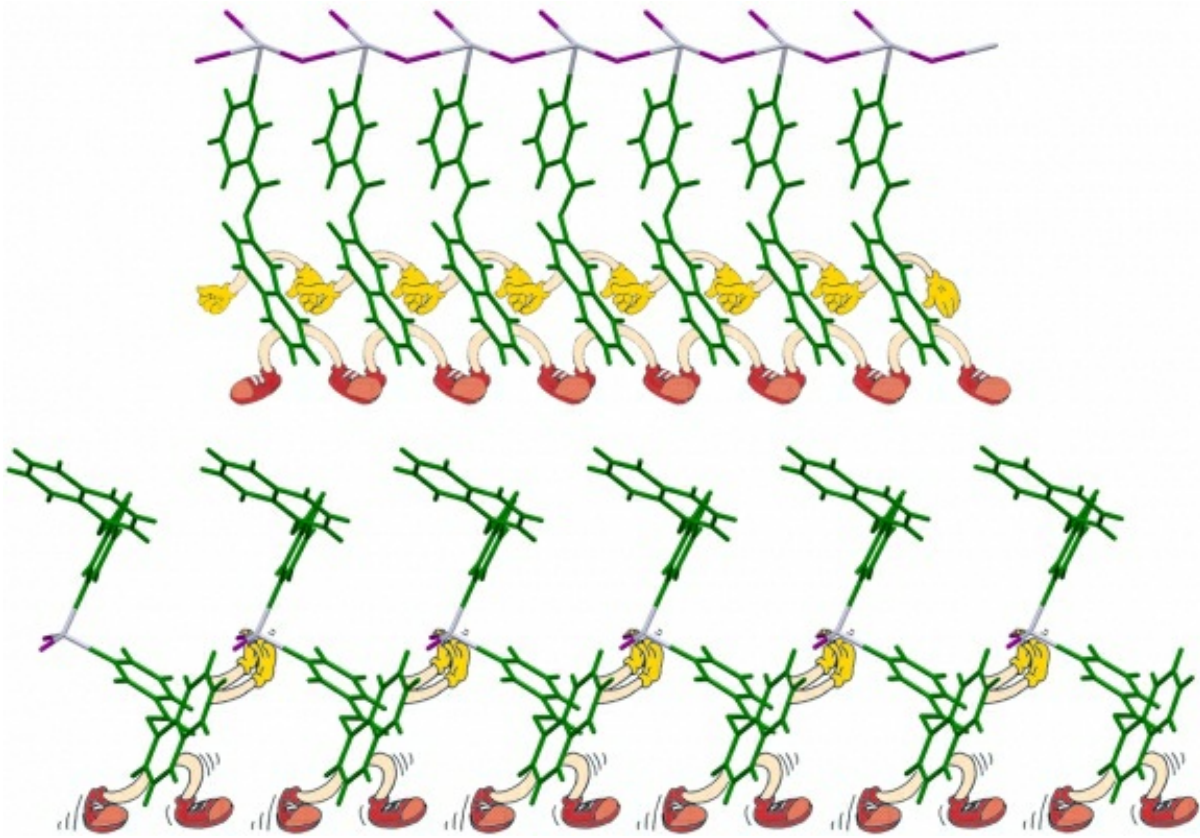

Keywords: crystal engineering, coordination polymer, metal complexes 